\title{
Effects of Virtual Reality Horse Riding Simulator Training Using a Head-Mounted Display on Balance and Gait Functions in Children with Cerebral Palsy: A Preliminary Pilot Study
}

\author{
Hae Won Kim¹, Ki Seok Nam², Sung Min Son' \\ 'Department of Physical Therapy, College of Health Science, Cheongju Universitiy, Cheongju; ${ }^{2}$ Department of Physical Therapy, Yeungnam University \\ College, Daegu, Korea
}

Purpose: The purpose of this study was to investigate the effects of three-dimensional virtual reality horse riding simulator training using a head-mounted display on gait and balance in children with cerebral palsy.

Methods: Ten children with cerebral palsy were randomly assigned to the horse riding simulator (HRS) group $(n=5)$ or the horse riding simulator with virtual reality (HRSVR) group $(n=5)$. To evaluate balance, center of gravity $(C O G)$ sway velocity and total sway distance of each group were assessed using the Wii balance board, and gait speed and stride length of each group were assessed using a gait analysis system.

Results: Intra-group comparisons between pre- and post-intervention measures revealed that there were significant changes in all gait and balance variables such as stride length, gait velocity, COG sway velocity and COG sway distance in the HRSVR group ( $p<0.05)$. In the HRS group, there were significant changes in all variables except stride length $(p<0.05)$. In addition, inter-group comparisons showed significant differences between the two groups in stride length, gait velocity and COG sway distance except COG sway velocity $(p<0.05)$. Conclusion: The findings of this study suggest that horse riding simulator training combined with 3D virtual reality can be a new positive therapeutic approach for improving functional performance in children with cerebral palsy.

Keywords: Cerebral palsy, Virtual reality, Head-mount display, Horse riding

\section{INTRODUCTION}

Children with cerebral palsy have neurodevelopmental disorders due to non-progressive lesions occurring in the immature brain during the prenatal fetal term or in early infancy. ${ }^{1}$ As they grow, children with cerebral palsy exhibit symptoms of motor disorders due to the impairment of sensorimotor function caused by neurodevelopmental disorders, and primary deficits include spasticity, muscle weakness, reduced coordination and a loss of selective motor control. ${ }^{2}$ These primary conditions additionally generate secondary deficits such as muscle contracture and alignment deformities of children with cerebral palsy, leading to a reduction in the level of functional activity and difficulty in balance control and walking necessary for performing activities of daily living., ${ }^{3,4}$

Received Sep 4, 2019 Revised Oct 16, 2019

Accepted Oct 17, 2019

Corresponding author Sung Min Son

E-mailssm0417@hanmail.net
Various therapeutic approaches, such as virtual reality, treadmill training, robot-supported therapy and horseback riding simulator, have been used to improve balance and gait in children with cerebral palsy. ${ }^{5-10}$ Among diverse therapeutic approaches, hippotherapy is a training method employing the natural gait of the horse to provide motor and sensory input to children with cerebral palsy. ${ }^{11-14}$ However, hippotherapy using a real horse is not frequently used among the general population due to its accessibility and high costs. Therefore, to deal with such problems, a therapeutic approach using a horse riding simulator which produces movements similar to those of a horse has been recently used for various types of patients with central nervous system impairment such as cerebral palsy and stroke patients. ${ }^{5,15}$ Lee et al. ${ }^{16}$ examined the effects of horse riding simulator training on the functional ability in children with cere-

Copylight (C)2019 The Korean Society of Physical Therapy

This is an Open Access article distribute under the terms of the Creative Commons Attribution Non-commercial License (Http:// creativecommons.org/license/by-nc/4.o.) which permits unrestricted non-commercial use, distribution, and reproduction in any medium, provided the original work is properly cited. 
bral palsy, and reported that horse riding simulator training improved static and dynamic balance abilities in children with cerebral palsy. In addition, several previous studies have reported that horse riding simulator training led to an improvement in functional activities such as decreased spasticity and the improvement of postural control and gait in children with cerebral palsy. ${ }^{5,11,16,17}$

Virtual reality (VR) training uses computer software to track the user's motions and show them on a TV screen. In VR training, since the training machine can detect motions of not only healthy people but also people with various functional disorders, users can perform various game interactions. This is a training method that can provide both interest and ease of use unlike simple rehabilitation equipment. Several previous studies reported that training utilizing virtual reality had a positive impact on upper and lower extremity functions, postural control and functional gait ability in patients with central nervous system impairment such as stroke and cerebral palsy. ${ }^{8,18-20}$ However, VR training methods currently used in clinical practice reproduce the motions of the patient in a two-dimensional (2D) space using a screen or a TV monitor, placing limitations on vividness and immersion for the patient's motions. Recently, with the development of information technology, programs for reproducing three-dimensional (3D) virtual reality using a head-mounted display (HMD) have been developed to resolve the problems described above. $^{21}$

Until now, most previous studies have investigated the effectiveness of horse riding simulator training or 2D VR training for the improvement of functional activities such as balance and gait in children with cerebral palsy. No prior studies have examined the efficacy of horse riding simulator training combined with 3D virtual reality for functional improvement in children with cerebral palsy. Therefore, this study aimed to investigate the effects of 3D virtual reality horse riding simulator training using a head-mounted display on gait and balance in children with cerebral palsy.

\section{METHODS}

\section{Subjects}

This study was conducted with 10 children with spastic diplegic or hemiplegic cerebral palsy. Before the experiment, the purpose and procedures of the study were fully explained to the subjects and their guardians, and informed consent was obtained from them.
The inclusion criteria were as follows: 1) children with cerebral palsy aged 6 to 14 years without visual impairment, 2) children who can understand and follow oral instructions, 3) children diagnosed with spastic diplegia or hemiplegia by a specialist, 4) children with cerebral palsy classified as the gross motor function classification system (GMFC) Level III or IV who are able to maintain independent static sitting posture, 5) children who have not performed systematic horse riding simulator training or muscle strengthening exercise within the last 6 months, and 6) chidren with no problem in vestibular system.

\section{Procedure}

Ten children with cerebral palsy enrolled in this study were randomly assigned to either the horse riding simulator (HRS) group $(\mathrm{n}=5)$ or the horse riding simulator with virtual reality (HRSVR) group $(n=5)$. Children in the HRSVR group were provided with horse riding simulator training combined with virtual reality. Children in the HRS group only received horse riding simulator training. All subjects received the same neurological physical therapy during the intervention.

The horse riding simulator used in this study was a horse riding machine manufactured by Panasonic (JOBA, EU7805, Panasonic, Japan) that can simulate the movements of a real horse. The horse riding simulator used in this study was designed to implement an 8 -shaped movement by using 5 axes, and this movement is designed to enable the user to experience 3D movements (back-and-forth, side-to-side, and up-and-down movements) like the movements of a real horse. The movements of the horse riding simulator were produced using an embedded program of the machine. During horse riding simulator training, children were allowed to adjust their own postures and they were required to hold the handle using upper limbs. Subjects were instructed to maintain the tension of the neck and a sticker was attached at the eye level of the subjects so that they could fix their gaze on a specific location. While a child was using the horse riding simulator, the therapist continuously orally requested the child to correct his or her posture behind the child to prevent safety accidents and to get the child to extend the trunk as accurately as possible. In this study, a head-mounted display, NOON VR+ (FXGear, Korea), was used to employ 3D virtual reality. It provides virtual reality on a smartphone with a screen size of 4.7-5.7 inches, a Full HD $(1,920 \times 1,080)$ or higher screen resolution, 
and a Gyro sensor. NOON VR+ is equipped with anti-blue-light lens to reduce eye strain and fatigue and protect eyes from UV rays and harmful light. In addition, we used VRFIT (M2Me Co., Ltd., Korea) equipped with an IoT (Internet of Things) sensor to link the horse riding simulator to a head-mounted display (HMD). VRFIT has embedded sensors such as a 9-axis sensor, an acceleration sensor, a gyro sensor and a geomagnetic sensor, and its operating frequency is $0.3 \mathrm{~Hz}-3 \mathrm{~Hz}$. Its main function is to implement $3 \mathrm{D}$ virtual reality according to the actual movements of the exercise machine. Its communication distance is about $10 \mathrm{~m}$ and it is used by connecting it to a smartphone via Bluetooth. In this experiment, the VRFIT Stepper app that can be linked to VRFIT was used for observation. The image of 3D virtual reality on head-mount display was configured to move the landscape around at the speed of the horse riding equipment. The horse riding simulator training intervention was conducted for 20 minutes per day, twice a week, for 4 weeks. The Wii balance board and OptoGait were used to assess balance and gait ability before and after the intervention. If participants were complain of dizziness or abnormal symptoms during the training, participants were retrain after to take a break.

\section{Assessment tool}

The Wii balance board (Nintendo, Japan) was used to assess balance, and center of gravity (COG) sway velocity and total sway distance were measured for 30 seconds. The Wii balance board has a $50 \mathrm{~cm} \times 50 \mathrm{~cm}$ rectangular shape and it is used as an input device for home game consoles. COP data is continuously collected through load cells located at four corners and provided to a Bluetooth-connected computer. Balancia software ver. 2.0 (Mintosys, Korea), a program to analyze COP data, was used to analyze the data. The data of the COP assessed by the Wii Balance Board was analyzed by the Balancia program on a computer connected via Bluetooth. The analysis results show the moving distance and velocity along $\mathrm{X}$ and $\mathrm{Y}$ axes of the COP, left and right body weight distributions, slope 95\%, and area 95\%. All data were sampled at $100 \mathrm{~Hz}$ and extracted with a $10 \mathrm{~Hz}$ low-pass filter. Assessment was performed five times for each item and data analysis was conducted with the mean of three intermediate measurement values excluding the first and last measurement data.

In this study, a gait analysis system (OptoGait, Microgate S.r.l, Italy) was used to measure temporal and spatial parameters of gait.
The OptoGait is composed of a $1 \mathrm{~m}$ long transmission bar, two reception bars and a webcam (Logitech Webcam Pro 9,000, Logitech lnc., Swiss). Each bar has 96 light-emitting diodes (LEDs) installed at $1 \mathrm{~cm}$ intervals and transmits and receives infrared signals. The optical sensor transmits and receives signals at $1,000 \mathrm{~Hz}$ and collects information about temporal and spatial parameters of gait and gait patterns while a subject is walking between two parallel bars. The webcam was activated to collect accurate data and the values of variables related to gait were processed using the OptoGait (Version 1.5.0.0 MicrogateS.r.l, Italy) software program.

\section{Statistical analysis}

Statistical analysis of collected data was conducted using Windows SPSS 22.0 version. The general characteristics (age, height, and weight) of the subjects in each of the HRS and HRSVR groups were analyzed using descriptive statistics. The Wilcoxon Rank test was used to make intra-group comparisons before and after the intervention, and the Mann-Whitney U-test was used to compare the two groups before and after the intervention. The level of statistical significance was set at $\mathrm{p}<0.05$.

\section{RESULTS}

A total of 10 subjects were recruited and they were randomly assigned to one of the two groups, the HRS group $(n=5)$ and HRSVR group $(n=5)$. The general characteristics of the two groups are shown in Table 1. The intra-group comparison between pre- and post-intervention measures showed that there were significant differences in gait velocity, sway velocity and sway distance except for stride length in the HRS group $(\mathrm{p}<0.05)$. In comparison, the HRSVR group showed significant differences in all assessment scores of stride length, gait velocity, COG sway velocity and COG sway dis-

Table 1. The general characteristics of subjects

\begin{tabular}{lcc}
\hline & HRS group $(n=5)$ & HRSVR group $(n=5)$ \\
\hline Male/Female & $2 / 3$ & $2 / 3$ \\
SDCP/HCP & $3 / 2$ & $3 / 2$ \\
Age $(\mathrm{yr})$ & $9.40 \pm 2.07$ & $10.00 \pm 2.55$ \\
Height $(\mathrm{cm})$ & $119.40 \pm 11.17$ & $120.80 \pm 7.50$ \\
Weight $(\mathrm{kg})$ & $30.00 \pm 4.64$ & $31.00 \pm 4.12$
\end{tabular}

Values are presented as mean \pm standard deviation.

HRS: horse riding simulator, HRSVR: horse riding stimulator with virtual reality, SDCP: spastic diplegia cerebral palsy, HCP: hemiplegic cerebral palsy. 
Table 2. Comparison of balance and gait function in between the HRS and HRSVR group

\begin{tabular}{|c|c|c|c|c|}
\hline Parameter & & HRS group $(n=5)$ & HRSVR group $(n=5)$ & $\mathrm{p}$ \\
\hline \multirow[t]{4}{*}{ Gait Velocity (m/sec) } & Pre & $0.56 \pm 0.13$ & $0.53 \pm 0.17$ & \\
\hline & Post & $0.61 \pm 0.11$ & $0.78 \pm 0.17$ & \\
\hline & $\mathrm{p}$ & $0.04^{*}$ & $0.04^{*}$ & \\
\hline & Post-Pre & $0.05 \pm 0.04$ & $0.24 \pm 0.11$ & $0.01^{+}$ \\
\hline \multirow[t]{4}{*}{ Stride Length $(\mathrm{cm})$} & Pre & $73.36 \pm 11.08$ & $71.43 \pm 7.62$ & \\
\hline & Post & $75.45 \pm 11.79$ & $83.85 \pm 6.61$ & \\
\hline & $\mathrm{p}$ & 0.08 & $0.04 *$ & \\
\hline & Post-Pre & $2.10 \pm 2.10$ & $12.42 \pm 5.57$ & $0.01^{+}$ \\
\hline \multirow[t]{4}{*}{ COG_Velocity (cm/sec) } & Pre & $7.65 \pm .348$ & $7.27 \pm 3.41$ & \\
\hline & Post & $6.97 \pm 3.32$ & $5.92 \pm 2.81$ & \\
\hline & $\mathrm{p}$ & $0.04^{*}$ & $0.04^{*}$ & \\
\hline & Post-Pre & $-0.69 \pm 0.32$ & $-1.35 \pm 0.74$ & 0.01 \\
\hline \multirow[t]{4}{*}{ COG_Distance (mm) } & Pre & $217.50 \pm 90.64$ & $213.99 \pm 94.50$ & \\
\hline & Post & $197.80 \pm 86.44$ & $176.66 \pm 82.62^{*}$ & \\
\hline & $\mathrm{p}$ & $0.04^{*}$ & $0.04^{*}$ & \\
\hline & Post-Pre & $-19.30 \pm 6.88$ & $-39.33 \pm 9.94$ & $0.01^{+}$ \\
\hline
\end{tabular}

Values are presented as mean \pm standard deviation.

HRS: horse riding stimulator, HRSVR: horse riding stimulator with virtual reality.

*Significant difference between pre- and post-test $(p<0.05)$, ${ }^{+}$significant difference compared with the HRSHMD $(p<0.05)$.

tance $(\mathrm{p}<0.05)$. In addition, the inter-group comparison revealed significant differences between the two groups in stride length, gait velocity and COG sway distance except for COG sway velocity $(\mathrm{p}<0.05)$ (Table 2).

\section{DISCUSSION}

In this study, we investigated whether horse riding simulator training using 3D virtual reality is an effective treatment method to improve gait and balance in children with cerebral palsy. The assessment of gait and balance before and after the intervention revealed that the HRSVR group showed greater improvement in balance and gait compared to the HRS group. These results suggest that horse riding simulator training combined with virtual reality can lead to greater improvement in functional performance of children with cerebral palsy than horse riding simulator training without virtual reality.

In this study, the COG sway velocity and sway distance were decreased in both groups, indicating that horse riding simulator training is an effective intervention for improving balance ability. Several previous studies have reported that horse riding training is a positive intervention method for postural control and balance ability in various types of patients with CNS disorders, which supports the results of this study. ${ }^{5,15,22}$ Champagne et al..$^{22}$ reported that 10 -week horse riding training had a positive effect on fine motor precision, balance, and muscular strength in children with cerebral palsy. In addition, a study of adult stroke patients found that mechanical horse riding rehabilitation training improved balance ability in stroke patients. ${ }^{15}$ This findings suggest that horse riding training can be an effective intervention method for improving head and trunk control and equilibrium responses according to postural sway. ${ }^{23}$

In addition, gait velocity was increased in both groups, and stride length was also improved in the HRSVR group. These results are in agreement with several previous studies which also demonstrated that horse riding training is an effective intervention method for improving gait in children with cerebral palsy. A study by Kwon et al. ${ }^{24}$ recruited 32 children with cerebral palsy and reported that horse riding training resulted in an improvement in gait velocity and stride length in children with cerebral palsy. The exact mechanisms through which horse riding training improves gait function in children with cerebral palsy are still unknown. However, the improvement of gait function in children with cerebral palsy is believed to be due to factors such as the improvement in the movements of the pelvis, lumbar spine and hip joints and the reduction of abnormal spasticity. Ribeiro et al. demonstrated that horse riding training is an intervention method that can improve lower extremi- 
ty muscle activity in children with cerebral palsy. ${ }^{25}$ Another previous study reported that horse riding training can have a positive effect on gait ability through the improvement of pelvic movements. ${ }^{24}$ In addition, a study on the impact of horse riding training in children with cerebral palsy reported that horse riding training reduces muscle spasticity in lower limbs in children with cerebral palsy. ${ }^{26} \mathrm{~A}$ reduction in spasticity can lead to an improvement of gait ability in children with cerebral palsy. In this regard, Terebessy et al. ${ }^{27}$ reported that a decrease in spasticity of lower extremity muscles by botulinum toxin treatment led to the improvement of gait function in children with cerebral palsy.

As mentioned above, balance and gait of children with cerebral palsy improved in both the HRS and HRSVR groups. However, the HRSVR group showed a greater improvement than the HRS group, and inter-group comparisons also showed that there were significant differences between the two groups. So far, no studies have examined the effects of horse riding training combined with virtual reality on functional activities such as balance and gait. Thus, to our knowledge, there is not sufficient evidence of the effects of horse riding training combined with virtual reality. However, there are mechanisms in terms of which we can consider the way horse riding training combined with virtual reality can improve functional activities in children with cerebral palsy. The effects of horse riding training can be explained in part by previous studies which reported that application of VR horse riding training with a 3D HMD increased brain activity by stimulating the brain and triggered the motivation and interest of subjects. A functional magnetic resonance imaging (fMRI) study investigated the effects of VR intervention on reorganization of the brain cortex in stroke patients and demonstrated that VR training is an intervention method which can increase cerebral cortical activity in stroke patients. ${ }^{28}$ The findings of the fMRI study can be seen as the evidence that VR training can elevate the activity of the primary motor cortex and primary somatosensory cortex and can induce neuroplasticity through lateralization of the cerebral cortex. In addition, Saposnik et al. ${ }^{19}$ reported that VR training enhanced motor control and increased subjects' interest in the training, thereby increasing their motivations for motor learning. Lee et al. ${ }^{29}$ examined the effects of VR games with a HMD on the function of the affected upper limb in 12 stroke patients and found that various upper limb functions were improved. Although the study by Lee et al. [29] was conducted with stroke patients and evaluated not lower limb functions but upper limb functions, its results are consistent with those of this study in that Lee et al. [29] also demonstrated that VR training with a HMD can lead to functional improvement.

The findings of this study suggest that horse riding simulator training combined with $3 \mathrm{D}$ virtual reality can be a new positive therapeutic approach for improving functional performance in children with cerebral palsy. However, this study has some limitations that need to be taken into consideration. First, this study is a preliminary research and a small sample size makes it difficult to generalize the study findings. Second, activities performed at home or in other institutions in addition to the intervention of this study may have affected gait or balance of the subjects but it was not possible to control such confounding variables. Finally, only children with GMFC Level III or IV cerebral palsy were included in this study, so the results of this study cannot be generalized to children with physical functional limitations. Therefore, these limitations need to be addressed in future studies.

\section{REFERENCES}

1. Barrett RS, Lichtwark GA. Gross muscle morphology and structure in spastic cerebral palsy: a systematic review. Dev Med Child Neurol. 2010; 52(9):794-804.

2. Barber L, Hastings-Ison T, Baker R et al. Medial gastrocnemius muscle volume and fascicle length in children aged 2 to 5 years with cerebral palsy. Dev Med Child Neurol. 2011;53(6):543-8.

3. Huijing PA, Benard MR, Harlaar J et al. Movement within foot and ankle joint in children with spastic cerebral palsy: a 3-dimensional ultrasound analysis of medial gastrocnemius length with correction for effects of foot deformation. BMC Musculoskelet Disord. 2013;14:365.

4. Skaaret I, Steen H, Huse AB et al. Comparison of gait with and without ankle-foot orthoses after lower limb surgery in children with unilateral cerebral palsy. J Child Orthop. 2019;13(2):180-9.

5. Baik K, Byeun JK, Baek JK. The effects of horseback riding participation on the muscle tone and range of motion for children with spastic cerebral palsy. J Exerc Rehabil. 2014;10(5):265-70.

6. Chen Y, Garcia-Vergara S, Howard AM. Effect of a home-based virtual reality intervention for children with cerebral palsy using super pop VR evaluation metrics: A feasibility study. Rehabil Res Pract. 2015;2015: 812348.

7. Kim OY, Shin YK, Yoon YK et al. The effect of treadmill exercise on gait efficiency during overground walking in adults with cerebral palsy. Ann Rehabil Med. 2015;39(1):25-31.

8. Massetti T, da Silva TD, Crocetta TB et al. The clinical utility of virtual reality in neurorehabilitation: A systematic review. J Cent Nerv Syst Dis. 2018;10:1179573518813541. 
9. Sterba JA. Does horseback riding therapy or therapist-directed hippotherapy rehabilitate children with cerebral palsy? Dev Med Child Neurol. 2007;49(1):68-73.

10. Wu M, Kim J, Gaebler-Spira DJ et al. Robotic resistance treadmill training improves locomotor function in children with cerebral palsy: A randomized controlled pilot study. Arch Phys Med Rehabil. 2017;98(11): 2126-33.

11. Benda W, McGibbon NH, Grant KL. Improvements in muscle symmetry in children with cerebral palsy after equine-assisted therapy (hippotherapy). J Altern Complement Med. 2003;9(6):817-25.

12. Forssberg H, Hirschfeld H. Postural adjustments in sitting humans following external perturbations: muscle activity and kinematics. Exp Brain Res. 1994;97(3):515-27.

13. Hammer A, Nilsagard Y, Forsberg A et al. Evaluation of therapeutic riding (Sweden)/hippotherapy (United States). A single-subject experimental design study replicated in eleven patients with multiple sclerosis. Physiother Theory Pract. 2005;21(1):51-77.

14. McGibbon NH, Benda W, Duncan BR et al. Immediate and long-term effects of hippotherapy on symmetry of adductor muscle activity and functional ability in children with spastic cerebral palsy. Arch Phys Med Rehabil. 2009;90(6):966-74.

15. Han JY, Kim JM, Kim SK et al. Therapeutic effects of mechanical horseback riding on gait and balance ability in stroke patients. Ann Rehabil Med. 2012;36(6):762-7.

16. Lee CW, Kim SG, Na SS. The effects of hippotherapy and a horse riding simulator on the balance of children with cerebral palsy. J Phys Ther Sci. 2014;26(3):423-5.

17. Mutoh T, Mutoh T, Tsubone H et al. Effect of hippotherapy on gait symmetry in children with cerebral palsy: a pilot study. Clin Exp Pharmacol Physiol. 2019;46(5):506-9.

18. Mouawad MR, Doust CG, Max MD et al. Wii-based movement therapy to promote improved upper extremity function post-stroke: a pilot study. J Rehabil Med. 2011;43(6):527-33.
19. Saposnik G, Cohen LG, Mamdani M et al. Efficacy and safety of nonimmersive virtual reality exercising in stroke rehabilitation (EVREST): a randomised, multicentre, single-blind, controlled trial. Lancet Neurol. 2016;15(10):1019-27.

20. Kim YN, Lee DK. Effects of dance sports in virtual reality on balance, depression and ADL in stroke patients. J Korean Soc Phys Ther. 2013; 25(5):360-5.

21. Stepan K, Zeiger J, Hanchuk S et al. Immersive virtual reality as a teaching tool for neuroanatomy. Int Forum Allergy Rhinol. 2017;7(10):100613.

22. Champagne D, Corriveau H, Dugas C. Effect of hippotherapy on motor proficiency and function in children with cerebral palsy who walk. Phys Occup Ther Pediatr. 2017;37(1):51-63.

23. Liptak GS. Complementary and alternative therapies for cerebral palsy. Ment Retard Dev Disabil Res Rev. 2005;11:156-63.

24. Kwon JY, Chang HJ, Lee JY et al. Effects of hippotherapy on gait parameters in children with bilateral spastic cerebral palsy. Arch Phys Med Rehabil. 2011;92(5):774-9.

25. Ribeiro MF, Espindula AP, Lage JB et al. Analysis of the electromiographic activity of lower limb and motor function in hippotherapy practitioners with cerebral palsy. J Bodyw Mov Ther. 2019;23(1):39-47.

26. Alemdaroglu E, Yanikoglu I, Oken O et al. Horseback riding therapy in addition to conventional rehabilitation program decreases spasticity in children with cerebral palsy: a small sample study. Complement Ther Clin Pract. 2016;23:26-9.

27. Terebessy T, Domos G, Hever D et al. Botulinum toxin treatment in children with cerebral palsy. Orv Hetil. 2019;160(28):1105-11.

28. Merians AS, Tunik E, Adamovich SV. Virtual reality to maximize function for hand and arm rehabilitation: exploration of neural mechanisms. Stud Health Technol Inform. 2009;145:109-25.

29. Lee SH, Jung HY, Yun SJ et al. Upper extremity rehabilitation using fully immersive virtual reality games with a head mount display. A Feasibility Study. PM R. 2019. 